\title{
Verzeichnis der Bearbeiter der 3. Auflage
}

Professor Dr. Tim W. Dornis, Professor an der Leuphana Universität Lüneburg

Dr. Jan Ebersohl, Rechtsanwalt in München

Professor Dr. Jan Eichelberger, Professor an der Gottfried Wilhelm Leibniz Universität Hannover Jörn Feddersen, Richter am Bundesgerichtshof

Professor Dr. Jörg Fritzsche, Professor an der Universität Regensburg

Professor Dr. Jochen Glöckner, Professor an der Universität Konstanz

Dr. Marcus Grosch, Rechtsanwalt in Mannheim/München

Professor Dr. Axel Halfmeier, Professor an der Leuphana Universität Lüneburg

Professor Dr. Christian Heinze, Professor an der Ruprecht-Karls-Universität Heidelberg

Dr. Nadine Herrmann, Rechtsanwältin in Hamburg

Professor Dr. Franz Hofmann, Professor an der Friedrich-Alexander-Universität

Erlangen-Nürnberg

Professor Dr. Nadine Klass, Professorin an der Universität Mannheim

Dr. Mark Lerach, Richter am Landgericht Köln

Professor Dr. Eva Inés Obergfell, Professorin an der Humboldt-Universität zu Berlin

Professor Dr. Louis Pahlow, Professor an der Goethe-Universität Frankfurt am Main

Professor Dr. Karl-Nikolaus Peifer, Richter am Oberlandesgericht Köln, Professor an der Universität zu Köln

Professor Dr. Alexander Peukert, Professor an der Goethe-Universität Frankfurt am Main

Professor Dr. Dörte Poelzig, Professorin an der Universität Leipzig

Professor Dr. Benjamin Raue, Professor an der Universität Trier

Dr. Emil Schwippert, Vorsitzender Richter am Oberlandesgericht Köln a. D.

Dr. Guido Toussaint, Rechtsanwalt beim Bundesgerichtshof in Karlsruhe, Notar a. D.

Professor Dr. Dr. h.c. Martin Paul Waßmer, Professor an der Universität zu Köln

Dr. Marc Zain, Rechtsanwalt in Köln 